\title{
Pour une géographie de la saleté
}

\section{Danouta Liberski}

\section{Citer ce document / Cite this document :}

Liberski Danouta. Pour une géographie de la saleté. In: Journal des africanistes, 1989, tome 59, fascicule 1-2. pp. 39-62; http://www.persee.fr/doc/jafr_0399-0346_1989_num_59_1_2277

Document généré le 30/05/2016 


\title{
Résumé
}

Dans le prolongement des travaux de M. Douglas et de E. Leach sur la notion de l'interdit, l'auteur une lecture de la conception dogon de la souillure en exploitant le très riche corpus de textes produit, depuis M. Griaule, les différents ethnographes de cette ethnie. Conduite à travers mythes, et croyances, l'analyse de la notion de puni - catégorie fondamentale de la pensée religieuse dogon se condensent des images, des représentations et des idées relatives à ce que nous subsumons sous notion d'impureté - amène l'auteur à formuler l'hypothèse qu'en cette ethnie, la souillure remet en cause le lien territorial d'une partie ou de tous les membres d'un village dans le moment même où l'individu « impur » subit une modification particulière de son corps, une expérience de ses limites. en cause le lien territorial d'une partie ou de tous les membres d'un village dans le moment même où l'individu « impur » subit une modification particulière de son corps, une expérience de ses limites.

\begin{abstract}
Towards a geography of pollution (Dogon, Mali). Building on the work of M. Douglas and E. Leach on the concept of prohibition, the author proposes an interpretation of the Dogon concept of pollution based on the very rich literature produced by ethnographers since M. Griaule. Through an examination of myths, rites and beliefs, the author analyzes the notion of puru. This fundamental category of Dogon thought condenses images, representations and ideas corresponding to what Westerners perceive as " impurity ». This leads to the hypothesis that in this population, the relationship to territory of some or all of the village members is modified at the very moment the "impure " person is undergoing a particular change in his body, an experience of his own limits.
\end{abstract}


DANOUTA LIBERSKI

\section{Pour une géographie de la saleté*}

Il se tient dans la pensée religieuse dogon un discours complexe, tendu par l'opposition de deux catégories, celles de śms et de pùru ${ }^{1}$, qui ont été rendues, faute de meilleurs termes, par les notions du pur et de l'impur. Corrigeons immédiatement l'effet de cette traduction. Pour les Dogon, áms signifie « vivant » et leur sert à désigner la qualité que possèdent la plupart des êtres, des animaux et des choses. A ce terme s'oppose toujours celui de pùru, terme qui ne signifie pas la mort (il existe un autre mot pour dire celle-ci, yimu), mais dont le sens se situe bien quelque part dans l'orbe de la mort (advenir au pùru mène à la mort à plus ou moins brève échéance si l'on n'y remédie pas par un rite). Toutefois, on serait en droit de rendre pùru par impur si à l'idée de souillure et de miasme que renferme habituellement cette notion, on ajoute l'idée de perte, d'instabilité ou de dispersion des instances qui constituent la personne (Dieterlen $1947: 84$ ).

Les premiers ethnographes des Dogon étaient pleinement conscients de l'écart qui existait entre les catégories traduites et leur traduction, entre les termes j́mo et pùru et ceux de pur et d'impur. On admettra qu'il s'agissait pour les auteurs d'une commodité de langage dans le souci d'éviter la répétition de termes qui, s'ils sont conservés dans l'opacité de la langue vernaculaire, rendent bien des fois la lecture d'un texte ardue. On sait les difficultés et souvent même l'impossibilité pour l'ethnologue de rendre compte

*. Nous n'avons pas effectué d'enquêtes de terrain en pays dogon et les matériaux traités ici proviennent tous de la très riche littérature qui a été produite sur cette ethnie depuis les premières enquêtes de Marcel Griaule. Une première élaboration de ce travail fut faite en 1980, époque où nous n'avions pas encore commencé nos recherches en pays kasena (Burkina Faso). Il s'agissait pour nous de conduire une réflexion sur la notion de l'interdit, dans le prolongement des études stimulantes de M. Douglas (1967) et de E. Leach (1958 et 1964). Les enquêtes menées chez les Kasena depuis 1981 nous ont appris à reconnaître, en cette ethnie « voltaïque », une conception de la « souillure » qui, du strict point de vue ethnographique, est très différente de celle exposée ici. Toutefois, il nous est apparu que dans ces deux ethnies, une enquête rigoureuse sur la question de la « souillure » conduit inévitablement à s'interroger sur les relations que différents types de "segments sociaux " (clan, lignage...) entretiennent avec leurs territoires respectifs, mais aussi sur les procédures rituelles qu'ils mettent en œuvre pour retracer les limites des espaces « pollués ». En vue d'une analyse comparative ultérieure, il ne nous a pas semblé inutile de reprendre les réflexions que nous avait suscitées le fantastique corpus de textes sur les Dogon. Que les différents auteurs de ce corpus veuillent bien nous pardonner cette incursion en leur domaine. Qu'ils n'y voient pas autre chose que l'expression d'un hommage rendu à leurs patientes et minutieuses recherches.

1. Pour la transcription des termes vernaculaires, nous nous sommes référée au dictionnaire du dogon établi par G. Calame-Griaule (1968), sauf mention contraire indiquée par le terme mis entre guillemets. 
de catégories indigènes qui n'ont pas leur équivalent dans notre langue, et donc, dans notre pensée. Mais ce souci légitime de parler clair ne nous empêche pas de mettre en doute la possibilité même d'une utilisation « innocente » de notions telles que celles de pureté, d'impureté, de souillure, etc. Le moins que l'on puisse en dire, c'est que ce ne sont pas des notions neutres. Comme si ces mots n'étaient pas chargés d'un sens, n'étaient pas le lieu de connotations diverses - toutes prises dans le réseau des significations de la tradition judéo-chrétienne (du moins dans l'état actuel où nous les a livrées l'Histoire) ; comme si ces mots n'étaient pas « doués d'une mémoire seconde ", selon la belle expression de R. Barthes, que l'on serait bien en peine de retrouver ailleurs, en d'autres cultures. Si l'on se rappelle que le mot «souillure " dérive du mot de l'ancien français «soil » qui signifie "l'abîme de l'enfer ", on mesurera la distance entre notre notion de souillure et ce qui est en jeu, pour les Dogon, dans la catégorie du pùru.

Aux effets de la traduction s'ajoute une autre difficulté pour le lecteur lorsqu'il cherche à appréhender, au travers des textes, les catégories dogon de óms et de pùru. L'idée de "saleté » que contient assurément la notion de pùru se voit en effet, d'un texte à l'autre, tantôt complètement effacée du champs sémantique recouvert par la notion, tantôt anéantie dans sa spécificité par l'emploi imprécisé du terme de «souillure ».

Ces remarques faites, il serait faux de laisser croire que notre propos est d'aboutir à une appréhension du pùru dans laquelle n'entrerait d'aucune manière de l'«impureté » et de la "souillure ». Il n'y a aucun doute sur la nature du système : il s'agit bien là d'un de ces " systèmes de la souillure ", dont la définition minimale est qu'ils se construisent sur le maintien d'un écart entre le "propre » et le "sale » (de Heusch 1971 : préface à Douglas).

\section{LE « MÉCANISME DE L'IMPURETÉ »}

D'un homme qui a rompu un interdit de type totémique, les Dogon disent qu'il est devenu pùru. Il sent ses forces diminuer, la maladie n'est pas loin et, s'il n'y prend garde en allant consulter un devin qui lui prescrira le rite à accomplir, il est voué, plus ou moins rapidement, à la mort . Il existe, par ailleurs, des individus qui sont affectés du signe pùru en permanence. La catégorie du pùru fonde alors un partage de la société. Sur l'un des versants se tiennent les íneũ ómo, les « hommes vivants » (la majorité des Dogon) ; sur l'autre versant se tiennent les ineũ pùru (les « hommes impurs ») d'une part, les hommes et les femmes de certaines castes, d'autre part. Dans cette division de la société en deux classes d'hommes s'inscrit toujours une différence dans l'activité rituelle. Les íneũ pùru, ces « ni morts ni vivants " disent encore les Dogon (Griaule 1948, rééd. 1966 : 126), sont chargés pendant les funérailles de toutes les tâches qui concernent le cadavre : le laver, le vêtir, le transporter au cimetière. Lors de certains sacrifices effectués aux funérailles, ils sont les seuls à pouvoir manger la viande des animaux immolés. Par contre, ils seront exclus des sacrifices faits aux 
ancêtres qui sont, eux, óms, "vivants ». Ce sont encore les íneũ pùru qui sont seuls habilités à conduire les rituels concernant cette catégorie de défunts qui, par la manière dont ils sont morts, mettent en danger le village (noyés, foudroyés, femmes mortes enceintes, mortes en couche...). Ils sont également chargés de la construction et de la réparation de la maison réservée aux femmes menstruées. Enfin, c'est parmi eux que tous les soixante ans, lors de la cérémonie du Sigui, seront choisis les initiants, ólu barũ, futurs maîtres de la Société des masques ${ }^{2}$. Quant aux gens de castes, on sait qu'ils se définissent (du moins en Afrique de l'Ouest) par un rapport privilégié au travail d'une matière (fer, cuir, bois, fibres végétales, voix). Ces " hommes blancs » (íneũ pilu), comme les appellent parfois les Dogon (op. cit. : 81), sont placés sous le signe du pùru sinon toujours par leur personne du moins par l'activité qu'ils exercent.

A la liste des gens qui sont soumis au pùru, il faut ajouter ceux qui le sont temporairement à une étape importante de leur cycle de vie : les nouvelles accouchées, les nouveau-nés, les circoncis dont les blessures sont encore fraîches, les femmes menstruées, les veufs et les veuves. Dans cette même classe, on pourrait citer encore les défunts qui portent la marque du pùru durant toute la période où, selon les représentations de la mort dans cette population, leur "double $»^{3}$ erre parmi les vivants. Une autre catégorie d'individus momentanément pùru rassemblent les meurtriers (qu'ils aient ou non commis leur acte volontairement) et, dans une moindre mesure, les hommes qui ont tué un ennemi au cours d'un combat.

Mais le terme de pùru s'applique aussi à des choses. Ainsi, certaines substances comme la graine du fonio (sa variété rouge, Digitaria exilis) qui est l'objet d'un interdit majeur - tribal, pourrait-on dire - pour tous les íneú jms, les « hommes vivants »; ou encore certains phénomènes comme le fait pour un arbre de ne pas porter de fruit : il est pùru, disent les Dogon, et il faut procéder à des rites de fumigation. Enfin, la marque du pùru s'accroche également à ce qu'on appellera une catégorie de sangs écoulés : le flux des menstrues, les lochies de l'accouchement, le sang de la circoncision et de l'excision, le " deuxième sang » du sacrifice - celui qui s'écoule épais et noir, lorsque la victime est proche de sa fin - , le sang de la blessure (si elle a été provoquée par un autre, même involontairement) et, bien sûr, le sang du meutre ${ }^{4}$.

Cette diversité d'emploi de la notion s'accompagne d'une polymorphie des rituels. Il n'y a pas un mais des rites de «purification » : rite de fumi-

2. Le statut d'íne pùru se transmet par ce qu'on pourrait appeler "l'ancêtre formateur » (naní, litt. : il a mis au monde), c'est-à-dire l'ancêtre qui a présidé à la naissance de l'enfant et auquel l'enfant, par la suite, adressera des sacrifices (Dieterlen 1941 : chap. 4 et Calame-Griaule $1965: 35,47$ ). Le discours mythique fait remonter l'origine des ineũ puiru à l'enfant qui eut pour « ancêtre formateur " le premier homme mort (Griaule 1938: 69 sq.).

3. Il s'agit d'une des composantes de la personne (kikinu sáy). Dans les premiers textes (Griaule 1948, Dieterlen 1941), on retrouvera les termes de " double " ou "d'âme " pour désigner cet élément de la personne. Lorsque la notion de personne, telle que les Dogon la conçoivent, fut mieux connue, le terme d'" âme " apparu aux chercheurs tout à fait inadéquat (Calame-Griaule 1965, Dieterlen 1973).

4. Sur les différentes catégories de sang que distinguent les Dogon, cf. Dieterlen 1947 et Calame-Griaule $1965: 75-80$. 


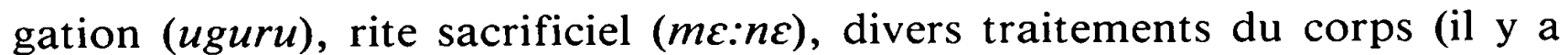
plusieurs termes pour désigner les rites cathartiques), rite dit kugudú effectué lorsque la terre et l'espace sont en jeu (une tortue d'eau est traînée autour du champ "gâté », ou dans les ruelles du village perturbé par le passage d'une femme menstruée, ou encore dans les pièces de la maison d'une femme morte enceinte).

Un texte de G. Dieterlen (1947: 81-90) sur le "Mécanisme de l'impureté chez les Dogon ", éclaire un pan essentiel de la notion de pùru. Il restitue minutieusement l'explication que formulent les Dogon lorsqu'ils s'interrogent sur le pourquoi et le comment de ces états (de maladie, de faiblesse, de malchance) provoqués par la rupture d'un interdit. L'exemple analysé par l'auteur est celui d'un homme qui a rompu un interdit de type totémique (il a tué, mangé ou simplement vu l'animal interdit de son clan). Cet homme, disent les Dogon, va perdre une partie de sa force vitale (nàma), cette force impersonnelle que tous les êtres et les choses « vivantes " (́mo) se partagent ${ }^{5}$. Ce départ crée un vide où viendra se loger la "force vitale » de l'animal totémique; force vitale qui, ainsi prise dans une composition étrangère, sera appréhendée comme une "saleté " (lógo, litt. : immondices, saletés). Le résultat est un mauvais mélange ; c'est, disent les Dogon, «le mélange de ce qui ne doit pas être mélangé ». L'être pùru est comme hétérogène en sa matière (on peut sans doute parler ici d'impureté, au sens chimique du terme).

Les rites qui visent à rétablir la personne dans son intégrité se dérouleront en deux temps ${ }^{6}:$ le temps du "nettoyage » de la saleté et le temps de la « réintégration » de la matière perdue. La catharsis sera prise en charge par un tiers. Celui-ci, après avoir fait mâcher à l'homme un peu d'écorce de cailcédrat, lui raclera la langue à l'aide d'un brin de balai neuf. Enfin, il lui balaiera la tête et le corps à l'aide d'un épi de mil qui, vidé par cette opération de son principe vital, devra être jeté hors du village.

Mâcher et recracher un peu d'écorce de caillcédrat constitue un préliminaire fréquent, une petite purification courante ${ }^{7}$. Cet acte et celui du raclage de la langue visent à chasser la "saleté » hors du corps. Ce serait là le premier temps. Le second temps serait celui du balayage avec l'épi de mil qui assure, dit-on, l'apport de la « substance régénératrice » (l'épi de mil en est comme "vidé ", on ne peut le replanter car il deviendrait mauvais).

Disons d'emblée que ce «mécanisme de l'impureté » met en jeu deux aspects de la notion de pùru: l'idée d'une perte et d'un vide, d'une part et, d'autre part, l'image d'une saleté. En outre, il vient nous dire comment,

5. Dans un texte ultérieur (Dieterlen 1973 : 204), il sera précisé que, conjointement à la perte de la "force vitale ", survient une déstabilisation et parfois un départ des huit symboles de graines cultivées inscrits dans les clavicules de tout homme « vivant».

6. Ce repérage, en réalité, ne fonctionne que pour les rites de purification d'une personne. Lorsqu'un lieu a été rendu purru, les rites effectués mettront en jeu des sacrifices ou l'abandon d'animaux vivants (tortue, rapace), et le repérage ci-dessus énoncé n'apparaît plus pertinent.

7. Il scmble que l'efficacité de l'écorce de caillcédrat est à comprendre en regard de l'amertume qu'elle dégage quand on la mâche. Le caïlcédrat est symboliquement lié à la vésicule biliaire qui mythiquement sera la base du premier médicament (Griaule et Dieterlen 1965: 294). 
très précisément, la « saleté » qui vient s'adjoindre au corps de l'homme est l'objet même que l'interdit maintenait à distance. La rupture d'interdit, en abolissant la distance, fait passer l'objet interdit d'un dehors à un dedans, sous la métonymie d'une "force vitale ${ }^{8}$. Autrement dit, l'interdit religieux est appréhendé, par les Dogon, comme ce qui instaure des limites dont la dissolution provoquée par la transgression a des effets sur le corps propre de l'individu. Nous reviendrons longuement sur ces notions de limites (du corps, du territoire) dans la seconde partie de notre article.

Cette conception " théorique " de l'impureté dogon n'offre cependant qu'un éclairage partiel de la notion de pùru. Il existe tout un pan de la réalité des faits, on l'a vu, que ne concerne en rien la rupture d'interdit, et qui trouve mal à s'expliquer par ce seul mécanisme. Il s'agit donc de voir si d'autres lectures de la notion de pùru sont possibles (plausibles).

\section{IMAGERIES}

Chacun sait que la notion d'impureté s'enfle des fantasmes qu'une société tient à l'endroit du corps. Un préalable s'impose donc, celui d'identifier ceux-ci avant de manipuler la notion de pùru. Les images seraientelles partout les mêmes?

On aura compris que le pùru entamait le corps à la fois comme perte de vitalité et comme «saleté ». Cependant, le texte de G. Dieterlen (1947) y insistait déjà, la notion de pùru serait avant tout subsumée sous la catégorie de l'incomplétude. Quand fut révélée aux chercheurs la fantastique version du mythe cosmogonique, celle du Renard pâle (Griaule et Dieterlen, 1965), la notion d'incomplétude de l'être pùru trouva une expression forte dans la figure d'une créature inachevée, Yourougou, le Renard pâle, dont l'imperfection fit advenir au monde une nouvelle qualité : l'impureté (Griaule et Dieterlen $1954: 86$ ). Rappelons, très brièvement, l'histoire de cette «imperfection ».

$\mathrm{Au}$ début du monde, dit le mythe, était un placenta divisé en quatre parties, chacune des parties contenant un couple de jumeaux mixtes, ou, plus exactement, les éléments mâles du couple étant déjà créés, ils attendaient leur jumelle. Dès ce moment, Ogo, l'un des jumeaux de la moitié inférieure du placenta, s'agite : il veut " voir " l'œuvre en cours d'Amma (nom donné à la divinité suprême). L'impatience dont Ogo fera preuve tout au long du récit lui vaudra, au terme de l'histoire, d'être transformé en yurugu (Vulpes pallida, le renard pâle). A s'en tenir au strict déroulement du mythe, l'incomplétude de l'être pùru est à lire dans cette figure du Renard. Ce n'est, en effet, qu'à cette ultime étape de son aventure que Ogo, circoncis et détaché de son placenta, effectuera une dernière descente sur la terre,

8. Nous n'avons cité qu'un seul exemple des interdits religieux qu'observe un individu. En réalité, ces interdits peuvent être alimentaires, sexuels, de lieu et de temps... Mais le texte de G. Dieterlen (1947) laisse penser que, dans ce mécanisme propre à la rupture d'un interdit religieux, il y avait à lire comme un paradigme. 
sera transformé en yurugu et adviendra au pùru. Privé de sa jumelle, privé de sa voix - il ne pourra " parler » qu'avec les traces que laissent ses pattes sur le sable (divination) - privé d'une fécondité « normale », le Renard est l'expression mythique de l'incomplétude.

Toutefois, cette idée de l'être pùru comme être « incomplet " n'épuise pas les fantasmes soutenus par la notion de pùru. Si l'on reprend le mythe, on constate tout d'abord que l'incomplétude de Ogo/Yourougou - telle qu'on peut la lire dans la diachronie du récit - est, en fait, l'histoire d'une perte. Pour avoir manifesté son désir d'ordre scopique, Ogo aura la veine de la langue coupée : il en perdra une partie du timbre de sa voix (Griaule et Dieterlen 1965: 179). S'arrachant trop tôt au placenta primordial d'Amma, il lui manquera, à jamais, sa jumelle et n'aura qu'une mauvaise fécondité : de ses rapports avec la terre (un morceau de son propre placenta, emporté dans la fuite) naîtront les génies chtoniens Yéban, uniques, monstrueux et incestueux (op. cit. : 180,185 et 187). Plus tard, dans une ultime tentative de combler son manque, il vole les "âmes » de sexe de son jumeau Nommo et recueille dans sa bouche une partie de sa semence (op. cit. : 244). Il aura le prépuce arraché, la langue déchirée et les dents cassées (op. cit. : 245). Il perd jusqu'à l'inscription somatique de sa jumelle (le prépuce ${ }^{9}$ ), est privé de sa voix et frappé d'impuissance (op. cit. : $246 \mathrm{sq}$ ). Enfin, transformé en yurugu, il ne récupère que deux de ses quatre «âmes» du sexe ${ }^{10}$ et une pseudo-fécondité : il engendrera, mais des animaux et un seul à la fois, ce qui, en regard de la règle de gémelliparité prévue par Amma pour tous les êtres, était une forme de stérilité (op. cit. : 267, 268). De transgressions en transgressions Ogo/Yourougou est donc gagné au manque et à la masculinité. Ainsi lue, l'histoire raconte plus que la perte d'une jumelle. C'est à proprement parler, la procréation qui échappe à Ogo (elle ne lui revient que mutilée).

On retrouve cette hantise dans la notion de pùru. Nous avions indiqué précédemment que la perte de "force vitale » subie par l'homme qui a rompu un interdit est solidaire d'un vide. Dans le rite que nous avions décrit, l'épi de mil, dont la substance régénératrice va combler ce "vide " en l'homme, est, à son tour, "vidé » de son principe vital; il ne pourra se reproduire, on le jette en dehors des limites du village. C'est un même type d'image qui est sollicité par les Dogon lorsqu'ils commentent l'interdit fait aux femmes menstruées (comme à d'autres catégories d'individus marqués par le pùru) de pénétrer dans le village. L'analyse des représentations liées à cet interdit, ainsi que l'analyse du rite de "purification » qui est accompli quand cet interdit a été rompu nous permettront de comprendre plus aisément quelle est la nature de cette «perte » et de ce « vide » dont l'être pùru est le siège.

Advenir au pùru pour une femme qui a ses règles, cela se traduit, pour elle, tout d'abord, par un déménagement : elle doit quitter sa maison, étein-

9. L.e prépuce est, pour l'homme, le support de sa féminité, comme le clitoris est, pour la femme, le support de sa masculinité (Griaule 1948, rééd. $1966: 148$ ).

10. L'être humain possèdera quatre " âmes de sexe " (kikínu) qui sont lićcs à sa procréation (CalameGriaule 1965: 37 et Dieterlen 1973: 207). 
dre son feu de cuisine, laisser là les outils quotidiens du ménage, pour aller habiter, en bordure du village, dans la "maison des femmes en règles ": Pendant la semaine (de cinq jours) où elle y restera, l'accès au village lui est absolument interdit. Son passage provoquerait des bouleversements graves et exigerait de nombreux rites visant non seulement les lieux où auraient porté ses pas, mais aussi tous les autels et toute l'eau du village (Paulme $1940: 263-8$ ). Cette eau que le village protège est celle contenue dans la grande jarre que chaque maison possède (et, par extension, toute eau contenue dans le moindre des récipients), mais c'est aussi l'eau des mares réservées à chaque famille indivise et encore celle de certains torrents et points d'eau « sacrés » (Dieterlen et de Ganay 1942 : 17-20). Protéger cette eau du village est, pour les Dogon, la raison principale de la mise à l'écart de la femme qui a ses règles ${ }^{11}$. L'interdit qui éloigne celle-ci du village repose, en effet, sur l'idée d'une extrême contagiosité du pùru. Deux types d'explications permettent aux Dogon de rendre compte de cet aspect du pùru. Le premier, qui nous est le plus familier, s'énonce en termes de propagation d'une "saleté ». La force vitale de la femme menstruée, porteuse de la " saleté » de cette dernière, vient contaminer l'eau. Cette eau contaminera, à son tour, tous ceux qui prendraient le risque de la boire. Le second type d'explication avancée par les Dogon repose, lui, sur une certaine idée de la « viduité » et de la « plénitude ». Ce deuxième type d'imagerie doit retenir notre attention.

L'eau, chez les Dogon, n'est pas une eau vide : elle est pleine de la présence d'une créature dont la forme et la couleur sont un rappel métaphorique de l'eau qu'elle habite. Son nom l'indique encore : nòmo (litt. : celui qui donne à boire) ${ }^{12}$. Le Nommo est très précisément lié à la fertilité des champs, à la fécondité des femmes, à la vie (op. cit. : 15) ${ }^{13}$. Il a, entre autres particularités, celle d'être présent dans la moindre goutte d'eau : céleste ou terrestre, pluies, rivières, eau des jarres, de la calebasse et ainsi du reste (op. cit. : 5). C'est de cette créature que l'eau se vide à l'approche de la femme menstruée (ou de tout être pùru). Littéralement, le Nommo les fuit. "Les pas de la femme en état d'impureté, écrira Griaule, vident de toute vie les lieux qu'elle ne doit pas fouler » (1948, rééd. $1966: 138)$. Elle est, elle-même, le siège d'une perturbation semblable à celle qu'elle provoque

11. De l'eau du village, on tient également à distance d'autres catégories d'individus soumis au pùru comme le porteur de fonio rouge (céréale püru, objet d'un interdit rigoureux pour tous les « vivants ", j́ms), le porteur de beurre de karité ou de cuivre (bracelets, anneaux...), l'homme qui a rompu un interdit de clan, les nouvelles accouchées, les gens de certaines castes (griots, cordonniers), de même que toute personne revêtue d'un pagne teint à l'indigo (une technique de teinture réservée aux femmes de cordonniers). Enfin, pour certains points d'eau, les personnes chaussées de sandales de cuir ne pourront s'en approcher. Toutes ces personnes sont dites pùru pour le génie des eaux Nommo (Dieterlen et de Ganay $1942: 17-20$ ).

12. Par un effet de langue, nòmo signifie également "celui qui boit " (Calame-Griaule 1965: 404). Le Nommo peut, en effet, vider les gens de leur vie en buvant leur sang (les noyés appartiennent à la catégorie du pùru).

13. Dans la version savante du mythe cosmogonique, le Nommo sera sacrifié et ressuscité pour réparer les désordres que son jumeau Ogo a provoqués dans la création. Il occupe, dans la genèse du monde, le pôle positif, lié à l'humidité, la f́ćcondité, la lumière, l'ordre, la vie. Son rôle est celui d'un organisateur et d'un gestionnaire du monde des hommes. Le pôle négatif est, bien entendu, occupé par Ogo/Yourougou. 
dans le village : l'écoulement de son sang est perçu comme un trop-plein que " pousse du dedans une bile surabondante » (ibid.).

Qu'y a-t-il à lire dans cette image de la fuite du Nommo à l'approche des êtres pùru? De quels dangers ces derniers menacent-ils le village ? Un geste rituel de « réparation » nous fournit un début de réponse. Ce geste est celui des femmes qui vident et remplissent d'une nouvelle eau toutes les poteries de toutes les maisons du village quand, par mégarde ou inconscience, une femme qui a ses règles est passée par là (Paulme 1940 : 264). Ce geste de vider/remplir vient comme inscrire dans la réalité sensible l'état de "viduité » dans lequel la femme menstruée a mis le village et, dans le même temps, il inscrit comment les gens du village pallient cet effet. Mais ce geste, croit-on, vient dire encore autre chose.

L'eau qui emplit les poteries n'est pas n'importe quelle eau. Elle provient de la grande jarre que chaque maison possède et qui semble être la propriété du lignage agnatique du mari : elle ne doit jamais quitter la maison et la femme qui divorce ne peut l'emporter (Dieterlen et Calame-Griaule 1960 : 56-7). Cette grande jarre, dans laquelle, chaque jour, la femme verse l'eau puisée à la mare " à l'aide de poteries appelées toroy dĭ buzu, poteries dont l'eau déborde » (op. cit. : 56), on la nomme d'un terme (ljy) qui désigne aussi l'enveloppe (amnios) qui entoure le fœtus (Calame-Griaule 1968). Dire avec certitude ce que représente cette jarre, nous ne pouvons le faire, mais l'idée générale de fécondité qui s'attache à l'eau et à l'activité féminine de puiser l'eau ${ }^{14}$, les noms évocateurs des poteries et de la jarre, nous amènent à nous demander si, dans cette grande jarre attachée à chaque maison, quelque chose de la procréation du lignage n'est pas en jeu. On peut penser, dès lors, que dans ce geste qu'effectuent toutes les femmes du village après le passage de la femme menstruée, dans ce vider-emplir l'eau des poteries, il faut lire la nature du danger que représente, pour le village, l'état de «viduité » de la femme menstruée. Tout se passe comme si la présence de cette dernière au sein du village "vidait » de son efficacité l'eau contenue dans les poteries, « vidait » le village de sa possibilité future d'avoir des enfants, bref, comme si la femme menstruée remettait en question toute la procréation du village. Dans le mouvement même où le geste rituel offre à lire ce danger, il nous dit comment le village pare à celui-ci en opposant sa « plénitude » (emplir l'eau) à la « viduité » de la femme menstruée (vider l'eau).

Cette menace que fait peser la femme menstruée sur la procréation du village semble bien être celle que représente tout être pùru pour les êtres, les choses ou les lieux «vivants» (́́ms). Le geste rituel que nous venons de rapporter est, en effet, également effectué par toutes les femmes d'un clan quand un homme a rompu un interdit de type totémique et qu'il a négligé de procéder aux rites de « réparation » (de Ganay 1942:60). Par ailleurs, le passage dans certains champs « réservés » d'un homme porteur de fonio rouge (céréale pùru, rappelons-le), d'un cordonnier, d'un homme

14. Son rôle de pourvoyeuse d'eau et de nourriture est en liaison étroite avec l'acte de fécondité, car c'est là « une autre manière de donner et d'entretenir la vie » (Dieterlen et Calame-Griaule 1960:58). 
ayant rompu un interdit grave ou encore, bien entendu, de la femme menstruée, fera, disent les Dogon, péricliter le mil, mettra en cause la fertilité des champs et, dans certains cas, empêchera la pluie de tomber (Dieterlen 1947 : 86 et Paulme 1940 b : 70).

Nous indiquions précédemment qu'à côté de ce discours sur la perte et le manque, la notion de pùru soutenait un autre discours à l'endroit du corps, agencé cette fois autour de l'opposition du propre et du sale.

On se souvient comment les Dogon identifiaient la "saleté » de l'être devenu pùru à une force vitale (celle de l'objet interdit) prise dans une composition étrangère. Disons d'emblée que cette théorisation de la "saleté " en termes de matière déplacée n'épuise pas le discours qui, à travers la notion de pùru, se tient sur la saleté. Non moins présente, en effet, est l'imagerie liée à la hantise de ces irruptions d'organiques que sont la pourriture, le corps mort ou encore ces diverses sortes de sangs écoulés que nous avons énumérés ci-dessus. Tous ces phénomènes, nous l'avons dit, portent la marque du pùru. Mais plus encore : en parcourant les réseaux symboliques mis en place par le mythe du Renard pâle ou par d'autres formes de discours, on peut constater qu'à la catégorie de pùru est associée, à maintes reprises, la notion de pourriture (j̀ms, à ton bas).

Donnons-en quelques exemples. La figure mythique de l'être pùru qu'est le Renard possède, nous dit le mythe, une « voix pourrie » (Griaule et Dieterlen $1965: 267$ et Calame-Griaule $1965: 102)^{15}$. Certaines des transgressions qu'il commettra sous sa forme de jumeau céleste (Ogo) auront pour sanction la pourriture et, partant, le pùru. L'acte d'Ogo, jugé par les docteurs dogon comme le plus grave, est le vol des huit graines cultivées créées par Amma et leur ensemencement dans la Terre. Mû par son désir d'égaler Amma dans la création, Ogo agit dans la démesure : il tente de s'emparer de la « semence d'Amma » (les huit graines) et cherche à féconder une partie de lui-même (la Terre qui, rappelons-le, est née d'un morceau du placenta d'Ogo). Cette démesure entraînera une transformation de la Terre qui devint pùru, sèche et stérile (Griaule et Dieterlen 1965: 200 sq.). Pour dire cette transformation, le récit mythique va utiliser l'image d'un petit morceau de placenta pourrissant, celui-là même qu'aura prélevé Ogo sur son propre délivre pour recouvrir ses semis volés. La suite de l'histoire dira, une fois encore, l'existence d'un lien entre la pourriture et le pùru. C'est bien, en effet, parce qu'ils auront poussé dans la pourriture que les plants de fonio (l'unique graine restée en la possession d'Ogo) seront pùru. Telle est l'origine mythique de la variété de fonio aux grains rouges, objet d'un interdit rigoureux pour tous les « hommes vivants » (íneũ ómo). Prenons enfin un dernier exemple dans un tout autre registre : en examinant la classification des paroles que font les Dogon ${ }^{16}$, on apprendra que la femme menstruée est symboli-

15. La « voix pourrie » (mi : jmu) qualifie une émission nasale de la parole, soit provisoire (rhume), soit permanente (voix nasillarde). Certains instruments, au son nasillard, sont également dits avoir une "voix pourrie" (comme la flûte à anche battante des chevriers) [Calame-Griaule 1968].

16. Les Dogon ont élaboré une véritable théorie de la parole. Selon celle-ci, les discours et les tons sur lesquels ils sont énoncés (grave, faible, fort, " voix pourrie ») sont classés suivant l'état du sujet parlant (paroles de sagesse, de tristesse, de colère, parole des femmes en règles) [CalameGriaule 1965 : 52-7]. 
quement rattachée à une catégorie de parole, dont le ton est qualifié de « voix pourrie » (nous reviendrons sur cette détermination qui assigne la femme menstruée à l'espace du Renard).

Cette série d'associations symboliques qu'opère la pensée dogon entre le pùru et le pourri soulève un problème d'interprétation. On ne peut, en effet, soutenir l'idée que, pour les Dogon, tous les êtres et les choses soumis au pùru seraient, du même coup, marqués par la pourriture. Cette dernière notion, pourrions-nous dire en première approximation, appartient plus encore que les idées de perte, de vide et de saleté - à ce que certains linguistes appellent le champ de connotation d'un terme ${ }^{17}$. Une telle terminologie serait justifiée en cela que le « pourri » n'est effectivement pas un " élément permanent du sens du mot » pùru, mais elle a le défaut d'accorder aux associations données ci-dessus le statut de « valeurs subjectives variables ${ }^{18}$, ce qu'assurément on ne peut leur prêter. Pour donner à notre distinction une valeur opératoire, il faudrait donc s'autoriser à modifier quelque peu la définition classique que font les linguistes du concept de connotation et admettre, avec certains auteurs, que dans le champ de cette dernière peuvent aussi être comprises des associations d'idées qui sont fixées une fois pour toutes par la tradition ${ }^{19}$.

Certains éléments de la vie rituelle dogon (mais également du mythe) nous invitent à replacer ces rapprochements entre le pùru et le pourri dans un ensemble de représentations liées à la germination et à la fermentation. Un bref examen de ces représentations devrait nous permettre d'éclairer partiellement ce qui pousse les Dogon à évoquer ensemble, dans certains contextes, la putréfaction et l'état de pùru.

Nous prendrons pour point de départ de notre analyse, l'évocation par le mythe de l'essai d'agriculture entrepris par Ogo. Disons d'entrée de jeu que cette séquence du mythe offre, tout à la fois, l'image du déroulement droit des travaux des champs, tels qu'ils sont effectués dans le monde des hommes, et l'image d'actes se présentant comme la stricte inversion des gestes mythiques et rituels qui, dans cette ethnie, président à la mise en culture des terres. Ainsi, on veut voir dans le vol des graines par Ogo une image inversée de l'acte qu'accomplira le Nommo-Forgeron en donnant aux hommes les huit graines cultivées. Suite à ce vol, nous dit le récit, Amma retirera les " âmes femelles » des graines volées. Les représentations liées aux rites agraires mettent en jeu, elles, l'idée qu'Amma, au temps des semailles, octroie aux graines cultivées leurs âmes femelles (à la fin des récoltes, des rites seront effectués afin de renvoyer les " âmes » des graines en leur lieu d'origine céleste). Enfin, le mythe précise que le morceau de placenta dont Ogo recouvrit ses semis fut écrasé (nàma) par le pied droit de l'émis-

17. Rappclons qu'on appelle connotation d'un mot « l'ensemble des caractères définissant un terme à l'exclusion des traits distinctifs suffisants constituant sa définition décisoire " (Grand Larousse de la langue française 1978).

18. Selon la définition de la dénotation (Grand Larousse de la langue française 1978).

19. Citons Roland Barthes : «Et dès lors que l'on quitte le dictionnaire des mots pour une table des sens culturels, des associations d'idées, bref pour une encyclopédie des idées reçues, on entre dans le champ infini des connotations " (1982:135). 
saire d'Amma (geste qui eut pour conséquence le pourrissement du placenta). Le cultivateur dogon, lui, lorsqu'il sème, tasse (nàma) la terre de son pied gauche (Griaule et Dieterlen 1965 : 208, 441-3).

Dans ce contexte, on ne peut manquer de dire que la pousse dans la pourriture des plants de fonio, entraînant la stérilité de la terre, est une image qui, dans le récit mythique, fonctionne comme antithèse de la germination des graines dans la fumure des champs. Un fait rituel éclaire ce tableau final du récit relatant la première mise en culture de la Terre et permet d'y voir un autre trait d'opposition à l'agriculture des hommes.

Comme dans de nombreuses sociétés d'Afrique de l'Ouest, les Dogon réservent au délivre d'un nouveau-né un traitement rituel complexe. Après la coupure du cordon ombilical, le délivre sera déposé dans une poterie pleine d'eau. Cette poterie devra ensuite être placée sous le fumier de tiges de mil que chaque maison possède. Considéré comme le jumeau de l'enfant, le placenta, ainsi replacé dans "l'eau de la mare ", est maintenu symboliquement «vivant » (́́ms). Les Dogon y insistent : bien que séparées d'avec l'enfant, les enveloppes placentaires ne peuvent être vouées à la pourriture qui désagrège les corps morts, elles ne peuvent être marquées par le pùru. Avec le temps, la poterie enfouie sous les tiges de mil en décomposition finira par se casser. Le placenta sera mêlé au fumier et contribuera à la fertilisation du sol et au renouveau des récoltes (Calame-Griaule 1965 : 293).

En projetant sur le fragment de mythe rapporté ci-dessus la lumière du rite, on voit donc prendre corps un nouveau paradigme. Il apparaît, en effet, que la notion d'un placenta mythique marqué tout à la fois par la pourriture et le pùru n'a de sens, dans la pensée dogon, qu'en regard d'un placenta qui, hors mythe, est associé à la fumure des terres et maintenu sous le signe du «vivant ". Les commentaires que font certains informateurs dogon à propos du processus «germinatif » de la création du monde, confirment l'existence d'un tel paradigme et permettent de préciser la nature de l'opposition en jeu. La germination des graines dans le placenta cosmique, au temps de la seconde genèse ${ }^{20}$ fut rendue possible, nous disent ces informateurs, par la " parole » (la vie) qu'Amma plaça en toutes graines. L'activité de cette « parole », de cette «vie », ajoutent-ils, doit être comparée au procès de la fermentation : c'est la fermentation dans le sein d'Amma qui provoqua l'éclatement et la germination des graines, ouvrant ainsi le monde et permettant son développement (Griaule et Dieterlen 1965 : 128). Ces représentations, confrontées avec l'image élaborée par le mythe de graines de fonio prenant vie dans un placenta en putréfaction, nous mettent en mesure d'affirmer que cette dernière image est construite sur la double opposition suivante : germination dans la pourriture/germination dans la fumure et placenta pourri/placenta qui fermente ${ }^{21}$.

20. Une première création du monde à partir de la graine d'acacia fut ratce, faute d'eau (Griaule et Dieterlen 1965 : 92).

21. «Dans le placenta d'Amma, dit-on, beaucoup de choses fermentaient» (Griaule et Dieterlen 1965 : 128). 
Ce long détour à travers mythe et rite nous aura fait entrevoir que les associations symboliques que font les Dogon entre le pourri et le pùru toujours rabattues sur l'axe de la stérilité - ne peuvent se comprendre qu'au regard de ces autres associations qui mettent en jeu, cette fois, la notion de " fermentation » et la catégorie de óms, l'une et l'autre étant toujours situées sur l'axe de la fécondité.

\section{LES NOMS PERDUS}

Nous avons, jusqu'à présent, cherché à repérer quels types d'imageries étaient mises en œuvre par la notion de pùru. Nous avons vu que derrière les idées de «perte » et de "saleté »- dimensions que mettait déjà en pleine lumière le texte de G. Dieterlen (1947) - se profilaient les fantasmes d'un corps vidé de son pouvoir de reproduction, d'une part, d'un corps voué à la décomposition stérile de la mort, d'autre part. Dans les pages qui suivent, notre souci sera différent. Au début de cet article, nous indiquions la diversité des emplois possibles du terme de pùru. Nous allons donc maintenant tenter de voir si, derrière cette diversité, il n'existe pas une détermination unique qui lierait ensemble tous les sèmes.

Des petits récits (mythiques ou autres) vont nous fournir un premier point d'appui pour cette seconde partie de l'enquête. Ces récits dévoilent un usage tout à fait remarquable du terme de pùru. Pour dire vite, la narration semble employer la catégorie de pùru comme une " technique " pour matérialiser des êtres invisibles ou, ce qui revient au même, pour faire changer d'espace des êtres, des animaux ou des choses. Nous avons sélectionné trois de ces récits.

\section{Récit mythique de l'une des origines du feu}

Un jeune chevrier qui gardait son troupeau entendit des coups insolites dont il ne put découvrir la cause. Revenu chez son père, il reçut de celui-ci le conseil de lancer des petits cailloux en direction du son. C'est ce que fit l'enfant et tout ce qu'il put toucher avec les pierres devint visible. Il aperçut alors plusieurs Forgerons du monde des Yébans assis autour d'un feu et frappant avec leur masse. Les Forgerons, rendus pùru pour les leurs - et, de ce fait, visibles - par le jet de pierre, furent capturés par le chevrier qui les emmena avec leurs outils et leur foyer dans sa famille qui se servit dès lors du fer et du feu (Griaule 1938: 49).

\section{Récit de la domestication des moutons}

Une jeune fille fut entraînée par un génie Nommo dans une rivière où on la chercha en vain. Peu de temps après, des femmes allant puiser, aperçurent la jeune fille alors qu'elle entrait dans l'eau suivie de deux moutons. Alertés, les gens du village guettaient son retour. Un jour, elle se laissa surprendre avant qu'elle ait pu disparaître dans l'eau avec les animaux. Les gens du village jetèrent sur elle de la cendre de fonio. Cette graminée étant l'interdit du Nommo, la jeune fille et les bêtes devinrent pùru pour le génie et ne purent retourner dans l'eau. La jeune fille fut ramenée au village avec les moutons qui, par la suite, se multiplièrent (Dieterlen et de Ganay 1942 : 9). 
Dans les deux récits, on le voit, le pùru inscrit son chiffre à l'intersection de deux espaces : espace des Génies-Forgerons/espace des hommes, dans le premier récit ; espace aquatique de la jeune femme/espace terrestre des hommes, dans le second. En outre, le pùru s'indique, à chaque fois, comme un rapport d'exclusion à l'un de ces espaces. Les Génies-Forgerons sont exclus de leur ancien monde invisible, ils surgissent dans l'espace humain, visible ; la jeune fille est exclue de son séjour aquatique chez le Nommo, elle advient à l'existence humaine, terrestre. Un troisième récit suggère un corrolaire à ces "définitions». Il relate l'apparition de la mort chez les hommes.

C'était au temps où la mort n'existait pas. Les hommes, lorsqu'ils étaient très vieux, se métamorphosaient en grand serpent et se rendaient dans le monde des génies Yéban. Les contacts avec les hommes n'étaient pas rompus : les serpents venaient, la nuit, prendre nourritures et boissons, dans les maisons des hommes. Mais il ne pouvait y avoir aucun contact direct, ni aucun échange de parole. Un vieillard, arrivé au temps de sa métamorphose, se transforma en serpent. Au même moment, des jeunes gens revêtus de jupes de fibres passaient par là. A cette vue, le vieillard déborda de colère et les invectiva en dogon. Il rompait là un interdit de parole. Devenu pùru pour les Yéban, il ne pouvait vivre avec eux. Son nouvel état, d'autre part, l'empêchait de retourner chez les hommes. Il mourut sur le champ (Griaule $1938: 65$ ).

Le vieil homme a mélangé sa parole de Dogon et son corps de génie. Pùru par la parole pour les Yéban, exclu par son corps du monde des hommes, le vieux dogon n'a plus de lieu où s'inscrire, il meurt faute d'espace. Cet emploi par les récits de la catégorie du pùru n'est pas seulement un effet littéraire. Cette " technique », si l'on peut dire, est utilisée dans la réalité par les ólu barú, ces jeunes gens choisis parmi les íneũ pùru pour devenir les initiés de la Société des masques ${ }^{22}$. Pendant le temps de leur retraite en brousse (temps de l'initiation), ils viennent rôder aux alentours du village et s'ils rencontrent des jeunes gens, ils leur lanceront des petits cailloux. Tous ceux qu'ils atteindront ainsi deviennent pùru pour le village et seront leurs captifs. Ils devront suivre les ólu barû́ dans leur retraite et les assister jusqu'à la fin de celle-ci (Griaule 1938: 189).

A l'évidence, ces faits (faits de discours et gestes rituels) indiquent qu'il y a, pour les Dogon, une relation entre la notion de pùru et l'idée d'un lien qui se rompt pour un être ou une chose appartenant à un espace déterminé. Peut-on repérer une relation de même type à chaque fois que les Dogon qualifient une personne ou un objet de pùru?

Lorsqu'on s'interroge sur le traitement rituel qui est réservé aux êtres pùru, un premier constat s'impose : pour la plupart de ces individus, l'appartenance au pùru - qu'elle soit temporaire ou permanente - se traduit par

22. Institution préposée au culte des morts. L'initiation des ólu barú a lieu théoriquement tous les soixante ans avant la grande cérémonie du Sigui dans laquelle les olù barū tiennent une place importante. Le Sigui est une fête rituelle interrégionale dont le " noyau » religieux est le culte du premier ancêtre mort sacrifié (Dyongou Sérou) [Griaule 1938, Leiris 1948]. 
une inscription géographique particulière ${ }^{23}$. Cette position réelle ou fantasmatique des êtres pùru est, selon les cas, l'intérieur du village, sa périphérie ou la brousse. Il y a là, croit-on, une véritable topologie à l'œuvre.

Nous avons déjà évoqué la femme menstruée qui, le temps de ses règles, est maintenue dans la "maison des femmes en règles ", à la périphérie du village. C'est là également que se tiennent les maisons des cordonniers et les cuves de teinture à l'indigo qu'utilisent leurs femmes. D. Paulme indique que les forgerons (les fondeurs) habitent des villages qui leur sont propres ou des quartiers séparés. Les forgerons isolés (qui travaillent uniquement le fer acheté aux premiers) habitent, eux, en bordure du village (1940:182).

C'est quelque part en dehors du village, en un lieu appréhendé comme " brousse », qu'a lieu l'opération de la circoncision. Un euphémisme éloquent qualifie l'opération de « sortie en brousse » (Calame-Griaule 1968). Les nouveaux circoncis restent sur le lieu de l'opération tant que la plaie saigne. Pendant tout le mois que dure leur retraite, ils vivent à l'écart du village, passant la journée sur les lieux où ils ont été circoncis, et la nuit dans la case qui, dans chaque quartier, est affectée aux circoncis du clan (Leiris et Schaeffner 1936). C'est plus loin dans la brousse, dans une caverne, que se déroulera l'initiation des ólu barú. Ils y vivront pendant trois mois, totalement séparés du milieu villageois. Pendant le temps de leur initiation, les villageois qui n'ont pas vécu un Sigui (c'est-à-dire, la majorité d'entre eux), ne pourront pas sortir du village. Si c'est l'époque des moissons, la récolte de leurs champs sera faite par les ólu barî́, qui garderont le produit de la vente. Ils sont, pendant trois mois, les véritables " maîtres de la brousse » (Griaule $1938: 184-90$ ).

Le meurtrier, lui, c'est dans un ailleurs sans nom qu'il erre, banni de la communauté des vivants et des morts. Par l'acte même où il fait couler du sang sur la terre, il a rompu quelque chose de son lien à l'espace du village $^{24}$. Dès cet instant, il est l'exclu. Un membre de sa famille, prévenu, viendra lui apporter la gourde et le bâton, symboles de sa destinée errante. Le meurtrier est bien d'un autre monde : si, par la suite, le hasard le met en présence d'un homme de sa famille, ils ne peuvent s'adresser la parole sans l'intermédiaire d'un tiers, un étranger, qui transmettra les questions et les réponses ${ }^{25}$. En regard des traitements que réservent aux meurtriers

23. Signalons que les ineũ pïru n'occupent pas de position géographique particulière, ils habitent et circulent dans le village. Par ailleurs, dans cette partie de l'article, nous n'envisagerons pas le cas des hommes qui ont rompu un interdit.

24. Sclon un ensemble de représentations liées au meurtre extrêmement répandues en Afrique de l'Oucst, la terre est "souillée " par le sang versé. Dès que possible, les parents du meurtrier et ceux de la victime se rendent ensemble sur les licux du meurtre. Le possesseur du champ égorgera un animal (bouf ou chère) fourni par les parents du meurtrier. Le sang de l'animal, en coulant au lieu même où fut versé le sang humain, " purifiera » la terre. Sur le meurtre et ses rites, voir la description précicuse (elle est unique) et fort détaillćc de D. Paulme (1940:116-23).

25. 1). Paulme (1940: 118) rapporte que le meurtricr peut parfois revenir au village (jamais avant trois ans). Mais la réintégration ne pourra avoir lieu qu'après un rite appelé « la requête par le taureau ". Le meurtricr doit se procurer un taureau à l'insu des Anciens du village. Il fera son entrée cans le village, les mains posées sur le corps de l'animal, qui est conduit par un membre de la famille du meurtrier (généralement son oncle paternel). Devant le conseil des Anciens il fera, par la bouche de son oncle, une requête qui n'est, paraît-il, jamais refusée. Le meurtrier, qui ne rompt 
d'autres populations de l'Afrique de l'Ouest (notamment certaines ethnies "voltaïques »), l'exclusion du meurtrier dogon apparaît radicale. Si, en ces ethnies (comme les Lobi, Dagari, Kasena), l'homicide provoque, comme chez les Dogon, une "souillure " de la terre et du meurtrier, les rites de "réparation » exigeaient rarement un bannissement à vie et, jamais, à notre connaissance, un effacement de la place qu'occupait le meurtrier dans sa généalogie (mort, un meurtrier pouvait, s'il avait des descendants, devenir un ancêtre). Un fait rapporté par D. Paulme permet peut-être de mieux comprendre le radicalisme des Dogon face à l'homicide. L'auteur indique, en effet, qu'avant la période coloniale, le mode de vie était tel que "le monde se limitait pour chacun au cercle étroit des parents et des alliés (...), tout étranger était considéré comme un ennemi "». Tuer un ennemi, chez les Dogon comme ailleurs, ne se range pas dans la même catégorie que le meurtre. Dès lors, si meurtre il y avait, la victime ne pouvait être qu'un parent ou un ami proche (Paulme 1940:116). Qu'il soit prémédité ou accidentel (la sanction était la même), l'acte présentait toujours « l'aspect monstrueux » (ibid.), d'une mise à mort d'un autre «soi-même ».

Tuer un ennemi, nous venons de le dire, n'était pas considéré comme un acte du même ordre que le meurtre. Le guerrier devait toutefois à son retour au village procéder à des rites de «purification » (de Ganay 1940 : 88-9). L'appartenance au pùru du guerrier met en jeu, une fois encore bien que d'une manière différente, un certain rapport à l'espace. L'homme qui avait tué un ennemi au cours d'un combat pouvait, à son retour, être pris de tremblements incoercibles. Cela allait parfois jusqu'à une sorte de folie au cours de laquelle il errait, çà et là, en brousse (de Ganay 1940 : 94). Il était, dit-on, « saisi » par le nàma (force vitale) de ses victimes qui, ainsi libéré par la mort, devenait pùru et "s'attaquait » à l'homicide comme à ses descendants (ibid.). Dès son retour au village, le guerrier devait faire un sacrifice à l'ancêtre protecteur de son clan. Mais, à son décès, la question se posait à nouveau de la protection de son fils ou de son futur « répondant »(nani :). Au moment de la cérémonie de levée de deuil, avant de poser la " poterie du mort » (dernière séquence des rites funéraires qui consacre le défunt comme ancêtre), le fils du défunt devait se rendre à la limite de la brousse et du village, en direction du lieu où son père avait tué, pour constituer un autel (une petite poterie fixée dans une masse de terre) appelé ólu gidu, littéralement «brousse fixée ${ }^{26}$. Les informations relatives à cette nécessité, à la mort d'un guerrier, de "fixer » quelque chose de la brousse sont peu claires quant à savoir ce qui vient là menacer le fils ou le "répondant » : l'ólu gídu est destiné à fixer "l'âme » du défunt mais, dit-on, c'est des victimes du défunt dont on se protège (op. cit. : 95). Quoi

jamais le contact d'avec le corps de l'animal, aide à ligoter et à coucher l'animal à terre. Dès que le sang de la gorge du taureau aura coulé sur la terre, l’homme est libéré de son bannissement, il pourra réintégrer l'espace du village.

26. Le terme désigne également les séparations en pierre ou en torchis qui divisent les champs el qui empêchent le lessivage des terres par l'écoulement des eaux de pluies (de Ganay 1940: 95). 
qu'il en soit, c'est encore les effets de l'acte d'homicide qui exigent une " réécriture " des espaces de brousse et de village.

Une autre catégorie d'êtres pùru à se voir assigner un espace différent est celle des morts. La mort, disent les Dogon, dissout le « lien qui retenait ensemble tous les éléments de la personnalité vivante " (Calame Griaule 1965 : 86). Les composantes de la personne (nàma, « force vitale ", kikínu, «composantes psychiques », graines claviculaires...), ainsi dispersées dans l'espace, deviennent éminemment dangereuses pour les vivants. Les rites funéraires et la cérémonie de levée de deuil (qui peut avoir lieu plusieurs années après le décès) auront pour visée d'assigner à ces composantes « dispersées 》 de nouvelles résidences (Dieterlen 1941 et Calame-Griaule 1965 : 86). Durant toute cette période, qui correspond en fait au temps du deuil, le défunt diton, erre entre brousse et village. Plus exactement dit, c'est l'une des « composantes psychiques » du défunt (kikínu sáy), sorte de «double » de la personne, qui, comme disent les Dogon, " se promène ». Quelques jours, voire trois ans avant la mort physique, les liens corps/« double » se relâchent : le kikínu sáy se détache, va en brousse, revient. Dès ce moment, l'homme appartient au pùru. Ce sera là son espace jusqu'à la cérémonie de levée de deuil. Cet espace du «mort » est, à proprement parler, un espace d'errance. La démarche du «double » est, dit-on, hésitante, incertaine, décrivant de grands zigzags, changeant brusquement de direction sans jamais aboutir nulle part (Calame-Griaule 1965: 87). La mort physique de l'homme va ramener son "double » au village. Il séjournera, un temps, dans la chambre du mort où se tient, reclus(e) la veuve ou le veuf. Une sorte de première levée de deuil, accomplie parfois bien avant la grande cérémonie de levée de deuil (Griaule 1938 : 389), chassera le "double " hors de la maison et le laissera, errant, aux abords du village où il sollicitera les vivants. Les rites de levée de deuil (damá) auront, entre autres buts, celui de rassembler les instances dispersées de la personne et de provoquer le départ définitif des kikínu pour le « pays des morts ». On pourra « poser la poterie »du mort, y effectuer des libations pour le «faire revivre »; le mort pourra avoir un "répondant " (nani :) chez les vivants, il n'appartient plus au pùru, il est un «ancêtre vivant » (́́ms).

Inversement à ce mouvement qui porte une partie des individus pùru à l'extérieur du village, les veufs et les veuves, d'une part, les nouvelles accouchées et les nouveau-nés, de l'autre, sont reclus au sein du village, dans une des pièces de leur maison. Cette réclusion prendra fin pour les veufs et les veuves avec la cérémonie de levée de deuil qui a lieu trois à quatre semaines après le décès d'une femme, de quelques mois à deux ou trois ans après le décès d'un homme. (La mention, par Griaule, d'un « damá pour mettre la veuve dans la maison " [1938: 344$]$ fait penser que la période de réclusion d'une veuve pouvait, en certains cas, être abrégée.) La femme qui vient d'accoucher et son nouveau-né ne sortiront de la pièce sombre où ils se tenaient depuis l'accouchement que sept semaines après la naissance de l'enfant (Dieterlen 1973 : 223). D'après D. Paulme (1940 : 438), la nouvelle accouchée, après sa réclusion, doit, à l'aide de feuilles de néré (Parkia biglobosa) qu'elle brûle, purifier la pièce où elle et l'enfant s'étaient tenus 
jusqu'alors. Et cela, ajoute l'auteur, afin d'apaiser et d'écarter les génies Yeban. La présence de ces petits êtres de brousse sur les lieux de l'accouchement et le geste rituel qui les en écarte sont pour nous des indices précieux. Ils illustrent cette " indétermination des limites qui séparent le village de la brousse " que met en lumière M. Cartry, à propos des Gourmantché du Burkina Faso (1979 : 265-88). Ils indiquent, en outre, que pour les Dogon, comme pour les Gourmantché, la venue au monde d'un nouvel être remet ces limites en question et nécessite de redessiner un espace de village au sein d'une " brousse " qui, au moment de l'accouchement, avait tout envahi (op. cit. : 277). Des gestes rituels d'un même type sont effectués au lendemain de la sortie de réclusion des veuves ou des veufs, dans la pièce où ils s'étaient tenus jusque-là (Dieterlen 1941 : 119 et Calame-Griaule 1965 : 128-9). La promiscuité de la mort à laquelle est soumis le conjoint survivant l'avait retranché de la vie sociale du village. Comme les êtres de brousse, le veuf ou la veuve s'étaient laissés pousser les cheveux ; ils avaient dormi à même le sol ; ils s'étaient nourris d'aliments peu agréables au goût (Dieterlen 1941 : 114). Le départ définitif du mort, en mettant fin à cette promiscuité, libère du même coup les survivants et les rend à l'espace du village. Tout se passe comme si la mort comme la naissance, en faisant irruption dans l'enceinte du village, abolissait les limites que le village oppose à la brousse, annulait les efforts des hommes qui, par le défrichement des terres, les semailles, les récoltes, cherchent à faire reculer la terre sèche et stérile du Renard ${ }^{27}$.

Le rapport d'exclusion d'avec l'espace du village qui signe l'appartenance des individus au pùru s'accompagne, pour certains, d'une perte momentanée de ce qui les distinguait comme êtres du village. Quand une personne est devenue pùru à la suite d'une rupture d'interdit, elle « perd ses noms et ses paroles » disent les Dogon (Calame-Griaule 1965 : 350). Elle perd les noms qui lui ont été imposés sept semaines après sa naissance par le patriarche du lignage ; imposition qui, au moment même où elle fut effectuée, fit sortir le nouveau-né de l'état pùru où il était depuis sa venue au monde ${ }^{28}$. D'autres, temporairement pùru, perdent leurs noms pour s'entendre appeler par « un nom commun qui désigne la catégorie à laquelle ils appartiennent " (ibid.). Le circoncis, tout le temps que dure sa retraite, sera "l'enfant de la brousse" (seli únum, en langue secrète). L'initiant à la Société des masques, l'ólu bárune, est, littéralement, « celui qui tient conférence en brousse » ou « qui est ajouté à la brousse »; il s'appellera ainsi pendant les trois mois de son initiation en brousse. En langue secrète, il

27. La brousse est mythiquement la terre du Renard. C'est un monde obscur, sec, stérile que le Renard parcourt en tous sens, sans repos; un monde lié à la masculinité de son hôte errant, un monde marqué par l'expérience de la pourriture et de la mort; bref, un monde entièrement sous la marque du püru. C'est sur cette terre que viendra se " plaquer " et s'étendre progressivement, au rythme de l'agriculture, la "terre pure " de l'arche du Nommo, venue sur la terre du Renard afin de la purifier, de la rendre $\mathbf{s} m$. , « vivante » et féconde. La "purification » est défrichement, scmailles, travaux agricoles: elle est ce que le village oppose à la brousse (Griaule et Dieterlen 1965).

28. Les quatre noms que déticnt tout individu renvoient à quatre aspects fondamentaux des relations sociales. Ils signent, chacun, les rapports qu'entretiendra un Dogon tout au long de sa vie avec, respectivement, son clan totémique, sa famille paternelle, sa famille maternelle et ses camarades de classe d'âge. (Pour l'analyse détaillée de ces noms propres, se reporter à Calame-Ciriaule 1965: 351-6.) 
sera appelé tána larani, « maître de la brousse 》 ou " usager de la brousse 》 (Leiris 1948 : 491). L'ine pùru n'aura d'autre nom que celui d'íne pùru pendant le temps où il s'occupera de la toilette et du transport des morts. Quant à la femme menstruée, s'il faut vraiment la désigner, on dira ya : pùnune, "femme qui a ses règles".

Le statut de ces êtres vacille, ils sont devenus des "gens du pùru", des «gens de la brousse ». La topologie et la sémantique se répondent, elles poussent les êtres pùru hors du village, elles leur assignent un autre lieu, la brousse. Quand on sait que les Dogon appellent " fils de la brousse " tout homme étranger au village (Leiris $1948: 199$ ), il ne fait plus aucun doute que c'est bien d'un territoire que sont exclus les êtres du pùru. La diversité des inscriptions spatiales des gens du pùru vient dire, pensons-nous, qu'il est différentes manières d'appartenir au pùru. Cette sorte de " géographie du pùru " n'est sans doute pas immédiatement cartographiable. Il faudrait, tout d'abord, forger les modèles graphiques qui permettraient de reporter sur un plan les associations brousse/gens du pùru qui sont établies par d'autres voies que le renvoi réel des individus à la brousse (nous pensons, notamment, au cordonnier, qui est une espèce de jumeau du Renard; ou encore aux íneũ pùru dont l'origine est liée à la mort de l'ancêtre Dyongou Sérou, le seul des quatre ancêtres apicaux à avoir pris le parti du Renard). Cela dit, le fait qu'une inscription spatiale singulière marque tout individu qui advient au pùru permet d'avancer que, pour les Dogon, il y a bien une corrélation entre l'état pùru où se trouvent, à un moment de leur existence, certaines personnes et la dissolution du lien qui rattache tout être "vivant » (j́ms) à l'espace du village. Comme les Forgerons-Yéban, ou la jeune fille des récits mythiques rapportés précédemment, les individus pùru « changent d'espace " mais, cette fois, pour prendre corps, si l'on peut dire, dans l'espace de la brousse, cette terre d'élection du Renard.

\section{DES LIEUX DE RUPTURE}

Que renvoie le village vers la brousse ? Quelle est la nature du danger dont il se sent menacé ? En quoi ce danger peut-il être identifié à l'espace du Renard ? En interrogeant, une fois encore, les représentations liées au sang des règles, il nous est apparu que l'on pouvait trouver sinon une réponse à ces questions, du moins les éléments nécessaires pour proposer ủne hypothèse.

Dans leur théorie de la parole, nous l'avons vu, les Dogon rangent le discours des femmes menstruées dans une catégorie (un «ton ») qualifié de "voix pourrie ». Trait remarquable, à cette catégorie s'accroche toujours un principe masculin ${ }^{29}$. Or, la règle qui préside à ce type de classifi-

29. La parole possède, à l'instar de l'homme, huit principes spirituels (kikinu). Ils se partagent de la même manière en quatre kikínu de corps (deux couples gémellaires mixtes, mâle et femelle) et quatre kikimu de sexe. Appliquée à la parole, la notion de kikimu désigne le con sur lequel la parole sexprime. Au ton " voix pourrie ", correspond le kikinu de sexe, mâle (Calame-Griaule $1965: 52$ sq.). 
cation veut que le sexe du sujet parlant et le sexe de sa parole correspondent. Que la femme menstruée puisse relever d'une catégorie de discours marquée par le masculin, cela, comme le soulignait déjà G. Calame-Griaule (1965 : 55), n'est, dès lors, pas loin de constituer une anomalie. Cette position singulière de la femme menstruée dans la classification des discours $a$, bien entendu, un sens. Pour l'entendre, il faut rappeler que la "voix pourrie » attribuée à la femme en règles nous renvoie au Renard qui, depuis ses dents cassées, ne possède plus qu'une voix nasillée, "pourrie ". Le Renard, à son tour, nous renvoie au sang des règles : c'est lui qui, selon l'une des versions du mythe (Griaule 1948, rééd. $1966: 20$ ), provoqua les premières menstrues en s'enfonçant dans le sein de la Terre. Les réseaux symboliques dogon y insistent : la femme menstruée est du côté du masculin, certes, mais dans cet espace stérile et sec que hante le Renard. Tout se passe comme si, pour les Dogon, la femme menstruée avait quitté l'espace féminin, humide et fécond, du Nommo - où son rôle de procréatrice la place - pour l'espace masculin, stérile et sec, du Renard - où son sang vide du germe que le Nommo dépose en toute femme (Dieterlen 1947 : 89) l'a poussée. On pourrait donc dire que l'arrêt momentané des fonctions procréatrices de la femme est pensé, par les Dogon, sur le mode d'une « rupture ", tant dans son habiter réel (elle déménage à la périphérie du village) que dans son habiter imaginaire (elle est identifiée au Renard).

Nous nous demandons si, à l'instar des représentations liées aux menstrues des femmes, ce n'est pas dans ces idées de différenciation d'avec un espace déterminé (celui de la procréation pour la femme menstruée) qu'il faut rechercher la nature du danger que réalisent les êtres du pùru. N'estce pas en ces lieux où les choses se différencient, où une " rupture " se produit (comme dans l'espace de travail des forgerons ou des artisans) que l'on repèrera la marque du pùru ? N'est-ce pas à chaque fois que quelque chose des limites du corps se fragilise, se modifie, se défait, que l'homme ou la femme bascule dans l'espace du pùru?

Dans les pages qui suivent, nous allons chercher à étayer cette hypothèse en analysant quelques cas de figure (nous ne pouvons, dans le cadre de cet article, envisager tous les lieux où le pùru porte sa marque).

La naissance est bien, pour les Dogon, de l'ordre de la rupture. Le nouveau-né, nous l'avons vu, est pùru jusqu'à l'imposition de ses noms, sept semaines après sa naissance. Du milieu aquatique d'où il vient (il nage dans le ventre de sa mère "comme un poisson ») au milieu terrestre où il choit (il y acquiert ce qui lui manquait, la « parole »), le nouveau-né a bien, fondamentalement, changé d'espace (Calame-Griaule 1965: 253 et Dieterlen $1973: 222$ ). Mais la véritable « rupture » se situe, pour les Dogon, dans la séparation de l'enfant d'avec le placenta, ce "petit frère », ce « jumeau » de l'enfant. Le traitement rituel qui vise à maintenir le placenta séparé toujours «vivant » (ómo) semble vouloir maintenir un lien ou, du moins, réduire l'écart qu'a creusé la coupure du cordon ombilical entre l'enfant et son jumeau. Il faut grandir et vivre, disent les Dogon, près de son placenta. Pendant sept semaines - chiffre de la pérennité - l'accouchée et le nouveau-né devront se laver sur la pierre plate qui recouvre la 
poterie contenant le délivre. L'eau sale de leurs corps s'écoule ainsi sur le contenu même de la poterie (Dieterlen 1973 : 223). Sept semaines après la naissance, l'enfant reçoit son premier nom qui lui est donné par le prêtre du clan de son père. Dans le moment même où l'enfant reçoit son nom et ses graines claviculaires, où il est intégré au clan de son père (il devient le « parent par le placenta » de tous les membres du clan), il cesse d'être pùru et devient śms, «vivant » (op. cit. : 224). Il est alors purolu, " hors d'atteinte du pùru " (Dieterlen 1941 : 208), jusqu'à la circoncision (ou l'excision), jusqu'à ce moment où quelque chose des limites de son corps va devoir, à nouveau, être rejoué.

On sait, depuis M. Griaule, que la circoncision (comme l'excision) est, pour les Dogon, la réduction nécessaire de l'ambivalence du jeune enfant. Porteur d'《 âmes » de sexe (kikínu) jumelles, mâle et femelle, il est encore un « poisson frais ", bisexué, non fixé dans l'appartenance à son sexe (Dieterlen 1973 : 222). Tant que le jeune garçon possède son prépuce, ce témoin de sa féminité (ou, pour une fille, son clitoris, témoin de sa masculinité), l'enfant est instable, " rien en lui n'est fixé ", et " ses rapports sont sans suite » (1948, rééd. $1966: 145$ sq). Les relations qu'entretiennent certains jeunes garçons, dès avant la circoncision, avec les fillettes, ne portent pas à conséquence : le non-circoncis n'est pas, pensent les Dogon, en mesure d'engendrer. La séparation d'avec son prépuce aura pour effet de verser le jeune enfant dans sa masculinité et de rendre ses rapports féconds; de même, l'excision fixera la jeune fille dans son identité sexuelle et lui permettra d'enfanter.

Cette séparation est le moment d'une rupture. Lors de l'opération de la circoncision, une simulation d'une seconde coupure du cordon ombilical, représenté par un petit bâtonnet posé sur la verge de l'enfant, est réalisée (Dieterlen $1966: 17)^{30}$. Pour la jeune fille, apparemment, rien de tel ${ }^{31}$. Mais les propos d'Ogotemmêli rapportés par $\mathbf{M}$. Griaule laissent entendre que tant la circoncision que l'excision se double de la coupure d'un lien de sang, d'un " fil de Dieu » qui, partant du prépuce ou clitoris et s'enfoncant dans le sol, attache tout enfant à la Terre depuis la naissance (Griaule 1948 , rééd. $1966: 149)^{32}$. Dans cette séparation symbolique d'avec la mère et/ou d'avec la terre, et dans cette perte nécessaire du prépuce ou clitoris,

:o. Il y a, dans le traitement des prépuces tranchés, quelque chose qui n'est pas sans ćvoquer le träatement du placenta séparé. Les prépuces seront enfouis sous le fumier du doyen du village et, comme le placenta, la perte ou le vol du prépuce (par un être animé de mauvaises intentions) auraient pour l'enfant des conséquences funcstes (Leiris et Schaeffner 1936 : 141-62).

31. Les informations sur le rite de l'excision sont moins nombreuses que celles relatives à la circoncision. A notre connaissance, il n'existe qu'une seule description de ce rite dans D. Paulme (1940: $486 \mathrm{sq}$ ). On y apprend, notamment, que tous les clans dogon ne pratiquaient pas l'excision de même que les gens de certaines castes. Sur le symbolisme de ce rite, on trouvera cependant de multiples précisions dans Griaule (1948), Griaule et Dieterlen (1965), Calame-Griaule (1965).

32. Attaché à la terre comme le Renard à son petit bout de placenta, l'enfant, comme le Renard, perd ce « fil de Dieu » dans le même temps où il perd son prépuce (ou clitoris). On dit du tout petit qu'il est mànu du Renard, son " parent à plaisanterie "; c'est, pour lui, un temps où il "voit " en brousse (notamment les « doubles » des morts). La circoncision et l'excision auraient-elles pour effet de détacher à jamais l'enfant du monde du Renard ? 
s'inscrit, aussi, pour l'enfant, une différence fondamentale dans son rapport avec le régime de la reproduction. Cette « seconde naissance » est aussi l'avènement à l'espace de la procréation. Et le nouvellement circoncis, comme la femme menstruée, saigne avant d'y accéder ${ }^{33}$.

Si la personne des individus castés n'est pas toujours sous le signe du pùru ${ }^{34}$, leur travail en revanche en possède bien le chiffre. Lorsqu'on cherche à le comprendre, c'est encore à ces notions de "rupture » et de " différenciation » que l'on est confronté, notions qui dans la pensée dogon président à toute naissance, à toute création. Ainsi, pour exemple, les forgerons semblent occuper, en regard de la catégorie du pùru, une position singulière. D'après $M$. Griaule, « la caractéristique des forgerons est une force vitale amoindrie, ce qui les éloigne de la catégorie des "vivants". Mais cet amoindrissement n'est pas comparable à celui de la mort, ce qui les éloigne aussi des "impurs". Ils ne sont pas comparables non plus aux " blancs" (les individus castés), car ceux-ci sont insensibles à certaines causes d'impureté " (1948, rééd. $1966: 81)$. Toutefois, la manipulation du fer et du feu qui fait advenir à l'existence des objets, la mutation que le forgeron fait subir aux matériaux est, pour les Dogon, assimilée à une œuvre de procréation et, comme telle, est, d'une certaine manière, pùru (Dieterlen 1966 : 17).

Une théorie du signe, extrêmement raffinée, éclaire ce danger que comportent l'acte sexuel, la procréation et, plus loin, la création d'objets. Au début du monde, dit le mythe, était le signe (búmõ). Le premier acte d'Amma fut de tracer en son sein - le «ventre de tous les signes du monde » - les 266 premiers signes qui préfiguraient le développement de tous les êtres et les choses de l'univers (Griaule et Dieterlen 1965:64 sq). Coextensif à la création de l'univers, le signe subit une modification et une progression dans sa forme. Il est d'abord trace (búmõ), l'idée de la chose ; puis marque (yàla), l'objet en pointillé ; ensuite schéma (tònu), l'objet prend corps ; et enfin dessin (tõ̃y), l'objet est créé, réel, achevé (Griaule et Dieterlen 1965 : $75 \mathrm{sq}$ ). Mais les Dogon savent la destinée mortelle du signe : de la trace de la chose à sa réalisation (son «dessin »), il y a ce commencement à être qui est le premier pas vers la destruction (op. cit. : 79). "Le dessin, disent les Dogon, est une chose qui a une fin » tandis que " le signe est le bon à venir, toujours là » (ibid.). En clair, pour les Dogon, " tout ce qui est sorti (né, créé) est un peu pùru » (Calame-Griaule 1965 : 201). C'est le risque nécessaire que prennent les hommes qui mettent au monde des êtres et des objets : ils les font advenir à l'existence, ils leur assignent une fin.

33. Les circoncis, comme les femmes, sont dits « en règles » tant que leur sang coulc. Leurs sangs sont similaires, pour les Dogon : celui de la circoncision élimine une «force mauvaise », celui des menstrues débarrasse la femme d'un « mauvais sang ». L'un comme l'autre sont nécessaires : ils précèdent le temps de la procréation.

34. La liste des castes appartenant au pùru n'est pas claire à nos yeux. Le texte du Renard pâle (1965) indique que seuls les cordonniers appartiennent au pùru. Les forgerons et les griots sont " différents " des Dogon, mais ils ne sont pas pùru. Par ailleurs, G. Dieterlen (1973) donne la liste suivante : les cordonniers, les griots, les fabricants de vannerie, les travailleurs du bois. D'après D. Paulme (1940), la fabrication d'objets en vannerie serait le fait des vieillards et celle d'objets en bois, le fait des forgerons. 
Ce que nous avons tenté de montrer, c'est que pùru désignait des lieux de rupture. Ces ruptures " incarnées » menacent le village dogon dans son intégrité spatiale, et c'est pourquoi on les rejette à la brousse et au Renard. Nous pensons d'autre part que le corps, chez les Dogon, ne se conçoit pas sans son appartenance à l'espace du village. Il est intégralement fixé à lui. Et c'est pourquoi, qu'il s'agisse du nouveau-né détaché du placenta ou du meurtrier qui, par son acte, a provoqué la mort d'un autre "soi-même ", on peut dire que ce qui légitime leur désignation pùru est une modification particulière de leur corps, une expérience de ses limites. Nous avons repéré deux « vecteurs " aux dispositifs que pùru nécessite. Un sens brousse et un sens village. Ainsi, les circoncis, les femmes menstruées, les jeunes initiés, les morts sont renvoyés à la brousse - le village s'en protège. Tandis que les veufs et les veuves, les accouchés et les nouveau-nés sont enfermés au sein du village. Le village prend le risque de les isoler à l'intérieur de ses limites pour les éloigner cette fois de la brousse, pour les en détacher.

On retiendra donc que tout le discours qui s'organise autour de l'opposition śms/pùru est soutenu par une idée du « vivant » comme corps lisse, bouché, voué à la complémentarité gemellaire, mais aussi en quelque sorte défini par l'espace du village, l'espace aménagé des habitations, des champs, des réseaux cultuels qui le parcourent. Ce corps-là est condamné à procréer, à produire. Toute faillite de ce projet, toute déchirure dans le corps vivant, dans l'enclos du village, est une brèche où revient la hantise du Renard. Car c'est le Renard qui, le premier, en déchirant son délivre, brisa l'unité d'Amma. Il donnait ainsi au monde des hommes l'indispensable différence, la redoutable différence.

Paris, CNRS, LA 221

Systèmes de pensée en Afrique noire

\section{BIBLIOGRAPHIE}

BARTHES, R. 1982. L'obvie et l'obtus. Essais critiques III. Paris, Seuil (« Tel Quel »), 282 p.

CALAME-GRIAUle, G. [1965], 1987. Ethnologie et langage. La parole chez les Dogon. Paris, Gallimard ; rééd. Paris, Institut d'ethnologie, avec une postface de l'auteur, $591 \mathrm{p}$.

- 1968. Dictionnaire dogon (dialecte toro). Langue et civilisation. Paris, Librairie C. Klincksieck, $329 \mathrm{p}$.

CARTRY, M. 1979. «Du village à la brousse » in M. Izard et P. Smith (éds.) : La fonction symbolique. Paris, Gallimard : 265-88.

DIETERlen, G. 1941. Les âmes des Dogon. Paris, Institut d'ethnologie, XL, 268 p.

- 1947. "Mécanisme de l'impureté chez les Dogon », Journal de la Société des africanistes, XVII : 81-90.

- 1952. "Classification des végétaux chez les Dogon », Journal de la Société des africanistes, XXII : 115-58.

- 1966. "Contribution à l'étude des forgerons en Afrique occidentale » in : Annuaire de l'EPHE 1965-1966. Paris, École pratique des hautes études, section des sciences religieuses : 5-28. 
- 1973. "L'image du corps et composantes de la personne chez les Dogon » in : La notion de personne en Afrique noire (Colloques internationaux du CNRS). Paris, CNRS : 205-29.

Dieterlen, G. et G. CALAME-Griaule 1960. "L'alimentation dogon », Cahiers d'études africaines, $3:$ : 46-89.

Dieterlen, G. et S. GANAY de, 1942. Le génie des eaux chez les Dogon. Miscellanea Africana, Lebaudy, 5, $48 \mathrm{p}$.

DOUglas, M. 1967. Purity and Danger. Londres. Routledge and Kegan Paul Ltd. (Traduction française sous le titre : De la souillure. Essai sur les notions de pollution et de tabou. Paris, Maspéro, 1971.)

GANAY, S. de, 1940. "Rôle protecteur de certaines peintures rupestres du Soudan français ", Journal de la Société des africanistes, X : 87-98.

- 1942. Le Binou Yebene. Miscellanea Africana, Lebaudy, 2, 62 p.

GRIAUle, M. 1938. Masques dogon. Paris, Institut d'ethnologie, 896 p.

- 1948. Dieu d'eau, entretiens avec Ogotemmêli. Paris, Éditions du Chêne (3e édition, Paris, Fayard, 1966, 222 p.).

GRIAUle, M. etG. DIETERLEN 1954. "The Dogon » in D. Forde (éd.) : African Worlds. Londres, Oxford University Press : 83-110.

- 1965. Le Renard pâle, tome 1: Le mythe cosmogonique, fasc. 1 : La création $d u$ monde. Paris, Institut d'ethnologie, $544 \mathrm{p}$.

HEUSCH, L. de, 1971. "Préface » in M. Douglas : De la souillure. Essai sur les notions de pollution et de tabou. Paris, Maspéro : 7-21.

LEACH, E. 1958. " Magical Hair », Journal of the Royal Anthropological Institute, vol. 88 : 147-64 (traduit sous le titre : "Cheveux, poils, magie » in E. Leach : L'unité de l'homme et autres essais. Paris, Gallimard, 1980 : 321-61).

- 1964. "Anthropological Aspects of Language : Animal Catégories and Verbal Abuse " in Eric H. Lenneberg (éd.) : New Directions in the Study of Language. Cambridge (Massachusetts), MIT Press (traduit sous le titre « Aspects anthropologiques de la langue : Injures et catégories d'animaux » in E. Leach : L'unité de l'homme et autres essais. Paris, Gallimard, 1980 : 263-97).

I.EIRIS, M. 1948. La langue secrète des Dogon de Sanga (Soudan français). Paris, Institut d'ethnologie, $530 \mathrm{p}$.

LEIRIS, M. et A. SCHAEFFNER 1936. "Les rites de circoncision des Dogon de Sanga ", Journal de la Société des africanistes, VI : 141-61.

PAUlME, D. 1940. Organisation sociale des Dogon. Paris, Éditions DomatMontchrestien, (Études de sociologie et d'ethnologie juridiques, XXXII), 603 p.

- 1940 b. "Sur quelques rites de purification des Dogon », Journal de la Société des africanistes, $\mathrm{X}: 65-78$.

POUR UNE GÉOGRAPHIE DE LA SALETÉ (DOGON, MALI)

D. LIBERSKI

Dans le prolongement des travaux de M. Douglas et de E. Leach sur la notion de l'interdit, l'auteur propose une lecture de la conception dogon de la souillure en exploitant le très riche corpus de textes qu'ont produit, depuis M. Griaule, les différents ethnographes de cette ethnie. Conduite à travers mythes, rites et croyances, l'analyse de la notion de puru - catégorie fondamentale de la pensée religieuse dogon où se condensent des images, des représentations et des idées relatives à ce que nous subsumons sous la notion d'impureté - amène l'auteur à formuler l'hypothèse qu'en cette ethnie, la souillure remet 
en cause le lien territorial d'une partie ou de tous les membres d'un village dans le moment même où 'individu "impur » subit une modification particulière de son corps, une expérience de ses limites.

\section{TOWARDS A GEOGRAPHY OF POLLUTION (DOGON, MALI) \\ D. LIBERSKI}

Building on the work of M. Douglas and E. Leach on the concept of prohibition, the author proroses an interpretation of the Dogon concept of pollution based on the very rich literature produced y ethnographers since M. Griaule. Through an examination of myths, rites and beliefs, the author analyzes the notion of puru. This fundamental category of Dogon thought condenses images, representations and ideas corresponding to what Westerners perceive as "impurity ". This leads to the hypothesis that in this population, the relationship to territory of some or all of the village members is modified at the very moment the "impure " person is undergoing a particular change in his body, an experience of his own limits. 